TAIWANESE JOURNAL OF MATHEMATICS

Vol. 16, No. 5, pp. 1719-1732, October 2012

This paper is available online at http://journal.taiwanmathsoc.org.tw

\title{
GENERALIZED INVEX SETS AND PREINVEX FUNCTIONS ON RIEMANNIAN MANIFOLDS
}

\author{
R. P. Agarwal*, I. Ahmad, Akhlad Iqbal and Shahid Ali
}

\begin{abstract}
In this paper, a geodesic $\alpha$-invex subset of a Riemannian manifold is introduced. Geodesic $\alpha$-invex and $\alpha$-preinvex functions on a geodesic $\alpha$-invex set with respect to particular maps are also defined. Further, we study the relationships between geodesic $\alpha$-invex and $\alpha$-preinvex functions on Riemannian manifolds. Some results of a non smooth geodesic $\alpha$-preinvex function are also discussed using proximal subdifferentiation. At the end, mean value inequality and the mean value theorem in $\alpha$-invexity analysis are extended to Cartan-Hadamard manifolds. Our results extend and generalize the known results in the literature.
\end{abstract}

\section{INTRODUCTION}

The notion of convexity plays an important and significant role in the theory of optimization. Since, convexity is often not enjoyed by real problems, various approaches have been proposed by several researchers in order to extend the validity of results to the larger classes of optimization. An important and significant generalization of convexity is invexity, which was introduced by Hanson [7] in 1981. Hanson's initial results inspired a great deal of subsequent work which has greatly expanded the roles and applications of invexity in nonlinear optimization and other branches of pure and applied sciences. Jeyakumar [8] and Noor [12, 13] have studied the properties of preinvex and $\alpha$-preinvex functions, respectively, and their roles in optimization and mathematical programming.

It has been found that few properties of convex functions are needed for estabilishing the results on Riemannian manifolds. Rapcsak [15] and Udriste [16] proposed a generalization which differs from the others in the use of a Riemannian manifold.

Received November 19, 2010, accepted November 23, 2011.

Communicated by Chong Li.

2010 Mathematics Subject Classification: 53C22, 58E10, 53B20.

Key words and phrases: Geodesic $\alpha$-Invex sets, $\alpha$-Invex functions, Geodesic $\alpha$-Preinvex functions, Riemannian manifolds.

*Corresponding author. 
In their setting, the linear space is replaced by Riemannian manifold and the line segment by a geodesic. Pini [14] introduced the notion of invex function on Riemannian manifold, while Mititelu [11] investigated its generalization. Ferrara and Mititelu [4] developed Mond-Weir type duality for vector programming problems on a differentiable manifold. Barani et al. [3] introduced the concepts of geodesic invex set and geodesic preinvex functions on Riemannian manifolds with respect to the particular maps. Recently, Li et al. [10] studied the weak sharp minima for constrained optimization problems on Riemannian manifolds and their characterizations. The methods involve appropriate tools of variational analysis and generalized differentiation on Riemannian and Hadamard manifolds.

In this paper, we define the concepts of geodesic $\alpha$-invex set and geodesic $\alpha$ preinvex function on Riemannian manifold. Using suitable conditions, some relations between geodesic $\alpha$-invex set and geodesic $\alpha$-preinvex function are established. We prove the existence condition for global minima of these functions by relaxing the smoothness condition on geodesic $\alpha$-preinvex functions and considering lower semicontinuity. At the end, we prove the mean value theorem for differentiable functions on $\alpha$-invex subsets of Riemannian manifolds which extends the results of Antczak [1] and Azagra et al. [2].

\section{Preliminaries}

First, we recall some basic definitions and known results about Riemannian manifolds. For the standard material on differential geometry, we refer to [9].

Let $M$ be a $C^{\infty}$ smooth manifold modelled on a Hilbert space $H$, either finite dimensional or infinite dimensional, endowed with a Riemannian metric $\langle,\rangle_{p}$ on the tangent space $T_{p} M \cong H$. The corresponding norm is denoted by \|\|$_{p}$ and the length of a piecewise $C^{1}$ curve $\gamma:[a, b] \rightarrow M$ is defined by

$$
L(\gamma):=\int_{a}^{b}\|\gamma \prime(t)\|_{\gamma(t)} d t
$$

For any two points $p, q \in M$, we define

$$
d(p, q):=\inf \left\{L(\gamma): \gamma \text { is a piecewise } C^{1} \text { curve joining } p \text { to } q\right\} .
$$

Then, $d$ is a distance which induces the original topology on $M$. We know that on every Riemannian manifold there exists exactly one covariant derivation called LeviCivita connection, denoted by $\nabla_{X} Y$, for any vector fields $X, Y \in M$. We also recall that a geodesic is a $C^{\infty}$ smooth path $\gamma$ whose tangent is parallel along the path $\gamma$, that is, $\gamma$ satisfies the equation $\nabla_{d \gamma(t) / d t} d \gamma(t) / d t=0$. Any path $\gamma$ joining $p$ and $q$ in $M$ such that $L(\gamma)=d(p, q)$ is a geodesic and is called a minimal geodesic. The existence theorem for ordinary differential equation implies that for every $v \in T M$, 
there exists an open interval $J(v)$ containing 0 and exactly one geodesic $\gamma_{v}: J(v) \rightarrow M$ with $d \gamma_{v}(0) / d t=v$. This implies that there is an open neighborhood $\bar{T} M$ of the submanifold $M$ of $T M$ such that for every $v \in \bar{T} M$, the geodesic $\gamma_{v}(t)$ is defined for $|t|<2$. The exponential mapping $\exp : \bar{T} M \rightarrow M$ is then defined as $\exp (v)=J_{v}(1)$ and the restriction of exp to a fiber $T_{p} M$ in $\bar{T} M$ is denoted by $\exp _{p}$ for every $p \in M$. We use parallel transport of vectors along geodesic. Recall that for a given curve $\gamma: I \rightarrow M$, a number $t_{0} \in I$ and a vector $v_{0} \in T_{\gamma\left(t_{0}\right)} M$, there exists exactly one parallel vector field $V(t)$ along $\gamma(t)$ such that $V\left(t_{0}\right)=v_{0}$. Moreover, the mapping defined by $v_{0} \mapsto V(t)$ is linear isometry between the tangent spaces $T_{\gamma\left(t_{0}\right)} M$ and $T_{\gamma(t)} M$, for each $t \in I$. We denote this mapping by $P_{t_{0}, \gamma}^{t}$ and we call it the parallel translation from $T_{\gamma\left(t_{0}\right)} M$ to $T_{\gamma(t)} M$ along the curve $\gamma$.

If $f$ is a differentiable map from the manifold $M$ to the manifold $N$, then $d f_{x}$, denotes the differential of $f$ at $x$. We also recall that a simply connected complete Riemannian manifold of nonpositive sectional curvature is called a Cartan-Hadamard manifold.

\section{Geodesic $\alpha$-Invex Sets And $\alpha$-Invex Functions}

In this section, we define geodesic $\alpha$-invex sets and $\alpha$-invex functions by introducing a bifunction $\alpha: M \times M \rightarrow R \backslash\{0\}$.

Definition 3.1. Let $M$ be a Riemannian manifold and $\eta: M \times M \rightarrow T M$ be a function, and $\alpha: M \times M \rightarrow R \backslash\{0\}$ be a bifunction such that for every $x, y \in M$, $\alpha(x, y) \eta(x, y) \in T_{y} M$. A non-empty subset $S$ of $M$ is said to be a geodesic $\alpha$-invex set with respect to $\eta$ and $\alpha$ if for every $x, y \in S$, there exists a unique geodesic $\gamma_{x, y}:[0,1] \rightarrow M$ such that

$$
\gamma_{x, y}(0)=y, \gamma_{x, y}^{\prime}(0)=\alpha(x, y) \eta(x, y), \gamma_{x, y}(t) \in S, \text { for all } t \in[0,1] .
$$

Remark 3.1. If $\alpha(x, y)=1$, then above definition reduces to that of geodesic invex set defined in [3].

We know that a subset $S$ of a Riemannian manifold is called geodesic convex if for any two points $x, y \in S$ there exists exactly one geodesic of length $d(x, y)$ which belongs entirely to $S$ (see $[9,16])$.

Remark 3.2. If we consider $M$ to be a Cartan-Hadamard manifold (either finite dimensional or infinite dimensional), then on $M$ there exists a natural map $\eta$ playing the role of $x-y$ in the Euclidean space $R^{n}$, for every $x, y \in R^{n}$. Indeed, we can define the function $\eta$ as

$$
\alpha(x, y) \eta(x, y):=\gamma_{x, y}^{\prime}(0), \text { for all } x, y \in M .
$$

where $\gamma_{x, y}$ is the unique minimal geodesic joining $y$ to $x$ (see [9, p.253]) as follows:

$$
\gamma_{x, y}(t):=\exp _{y}\left(t \alpha(x, y) \exp _{y}^{-1} x\right) \text {, for all } t \in[0,1] \text {. }
$$


Therefore, every geodesic convex set $S \subseteq M$ is a geodesic $\alpha$-invex set with respect to $\eta$ and $\alpha$ defined in $(*)$. Note that the converse is not true in general.

Example 3.1. Let $M$ be a Cartan-Hadamard manifold and $x_{0}, y_{0} \in M, x_{0} \neq y_{0}$. Let $B\left(x_{0}, r_{1}\right) \cap B\left(y_{0}, r_{2}\right)=\emptyset$ for some $0<r_{1}, r_{2}<\frac{1}{2} d\left(x_{0}, y_{0}\right)$, where $B(x, r)=$ $\{y \in M: d(x, y)<r\}$ is an open ball with centre $x$ and radius $r$. We define

$$
S:=B\left(x_{0}, r_{1}\right) \cup B\left(y_{0}, r_{2}\right) .
$$

Then, $S$ is not a geodesic convex set because every geodesic curve passing through $x_{0}$ and $y_{0}$ does not completely lie in $S$. Now we define the function $\eta: M \times M \rightarrow M$ such that

$$
\eta(x, y):= \begin{cases}\exp _{y}^{-1} x ; & x, y \in B\left(x_{0}, r_{1}\right) \text { or } x, y \in B\left(y_{0}, r_{2}\right) \\ 0_{y} ; & \text { otherwise. }\end{cases}
$$

For every $x, y \in M$, consider a bifunction $\alpha: M \times M \rightarrow R \backslash\{0\}$ and $\gamma:[0,1] \rightarrow M$ defined by

$$
\gamma_{x, y}(t):=\exp _{y}(t \alpha(x, y) \eta(x, y)), \text { for all } t \in[0,1] \text {. }
$$

Hence,

$$
\gamma_{x, y}(0)=y, \gamma_{x, y}^{\prime}(0)=\alpha(x, y) \eta(x, y) .
$$

Now, we show that $S$ is a geodesic $\alpha$-invex set with respect to $\eta$ and $\alpha$. Let $x, y \in$ $B\left(x_{0}, r_{1}\right)$, since $B\left(x_{0}, r_{1}\right)$ is geodesic convex (see [9, p.259]), it follows that,

$$
\gamma_{x, y}(t):=\exp _{y}\left(t \alpha(x, y) \exp _{y}^{-1} x\right) \in B\left(x_{0}, r_{1}\right) \subset S, \text { for all } t \in[0,1] .
$$

Similarly, for $x, y \in B\left(y_{0}, r_{2}\right)$, we have

$$
\gamma_{x, y}(t) \in S, \text { for all } t \in[0,1] .
$$

If $x \in B\left(x_{0}, r_{1}\right)$ and $y \in B\left(y_{0}, r_{2}\right)$ or $x \in B\left(y_{0}, r_{2}\right)$ and $y \in B\left(x_{0}, r_{1}\right)$ then, we have

$$
\gamma_{x, y}(t):=\exp _{y}\left(t \alpha(x, y) 0_{y}\right)=y \in S, \text { for all } t \in[0,1]
$$

Hence, $S$ is a geodesic $\alpha$-invex set with respect to $\eta$ and $\alpha$.

Let $\mathrm{S}$ be a geodesic convex subset of a finite dimensional Cartan-Hadamard manifold $M$ and $x \in M$. Then, there exists a unique point $p_{s}(x) \in S$ such that for each $y \in S, d\left(x, p_{s}(x)\right) \leq d(x, y)$. The point $p_{s}(x)$ is called the projection of $x$ onto $S$ (see $[6$, p. 262]).

Example 3.2. Let $S_{1}$ and $S_{2}$ be two nonempty closed geodesic convex subsets of a finite dimensional Cartan-Hadamard manifold $M$ such that $S_{1} \cap S_{2}=\emptyset$. Let us define 
$S:=S_{1} \cup S_{2}$ and $\eta: M \times M \rightarrow M$ by

$$
\eta(x, y):=\left\{\begin{array}{l}
\exp _{y}^{-1}\left(p_{S_{1}}(x)\right) ; y \in S_{1}, x \in M, \\
\exp _{y}^{-1}\left(p_{S_{2}}(x)\right) ; y \in S_{2}, x \in M, \\
0_{y} ; \text { otherwise. }
\end{array}\right.
$$

Now, for every $x, y \in S$ we define

$$
\gamma_{x, y}(t):=\exp _{y}(t \alpha(x, y) \eta(x, y)), \text { for all } t \in[0,1],
$$

we can see easily

$$
\gamma_{x, y}(0)=y, \gamma_{x, y}^{\prime}(0)=\alpha(x, y) \eta(x, y), \gamma_{x, y}(t) \in S, \text { for all } t \in[0,1] .
$$

Hence, $S$ is a geodesic $\alpha$-invex set with respect to $\eta$ and $\alpha$.

Now, we introduce $\alpha$-invexity and geodesic $\alpha$-preinvexity on geodesic $\alpha$-invex subset of a Riemannian manifold.

Definition 3.2. Let $M$ be a Riemannian manifold and $S$ be an open subset of $M$ which is geodesic $\alpha$-invex set with respect to $\eta: M \times M \rightarrow T M$ and $\alpha: M \times M \rightarrow$ $R \backslash\{0\}$. Let $f$ be a real differentiable function on $S$. Then, $f$ is said to be $\alpha$-invex with respect to $\eta$ and $\alpha$ on $S$ if

$$
f(x)-f(y) \geq d f_{y}(\alpha(x, y) \eta(x, y)) \text { for all } x, y \in S .
$$

Definition 3.3. Let $M$ be a Riemannian manifold and $S \subseteq M$ be a geodesic $\alpha$-invex set with respect to $\eta: M \times M \rightarrow T M$ and $\alpha: M \times M \rightarrow R \backslash\{0\}$. The function $f: S \rightarrow R$ is said to be geodesic $\alpha$-preinvex if for every $x, y \in S$, we have

$$
f\left(\gamma_{x, y}(t)\right) \leq t f(x)+(1-t) f(y), \text { for all } t \in[0,1],
$$

where $\gamma_{x, y}$ is the unique geodesic defined in Definition 3.1. If the above inequality is strict, then $f$ is called strictly geodesic $\alpha$-preinvex function.

Proposition 3.1. Let $M$ be a Riemannian manifold and $S \subseteq M$ be a geodesic $\alpha$-invex set with respect to $\eta: M \times M \rightarrow T M$ and $\alpha: M \times M \rightarrow R \backslash\{0\}$. Suppose that $f: S \rightarrow R$ is a geodesic $\alpha$-preinvex function, then every lower section of $f$ defined by

$$
S_{\lambda}:=\{x \in S: f(x) \leq \lambda\}, \lambda \in R,
$$

is a geodesic $\alpha$-invex set with respect to $\eta$ and $\alpha$.

Proof. Let $x, y \in S_{\lambda}$. Since $S$ is a geodesic $\alpha$-invex set with respect to $\eta$ and $\alpha$, there exists exactly one geodesic $\gamma_{x, y}:[0,1] \rightarrow M$ such that

$$
\gamma_{x, y}(0)=y, \gamma_{x, y}^{\prime}(0)=\alpha(x, y) \eta(x, y), \gamma_{x, y}(t) \in S, \text { for all } t \in[0,1] .
$$


By the geodesic $\alpha$-preinvexity of $f$, we have for all $t \in[0,1]$

$$
\begin{gathered}
f\left(\gamma_{x, y}(t)\right) \leq t f(x)+(1-t) f(y) \\
\leq t \lambda+(1-t) \lambda=\lambda .
\end{gathered}
$$

Therefore, $\gamma_{x, y}(t) \in S_{\lambda}$ for all $t \in[0,1]$, and the result is proved.

\section{Geodesic $\alpha$-PreinVeXity and Differentiability}

In this section, we introduce property and the condition (say condition (C)) on the function $\eta: M \times M \rightarrow T M$ and $\alpha: M \times M \rightarrow R \backslash\{0\}$ which will be use in the subsequent analysis.

Definition 4.1. (Property (P)). Let $M$ be a Riemannian manifold and $\gamma:[0,1] \rightarrow$ $M$ be a curve on $M$ such that $\gamma_{x, y}(0)=y$ and $\gamma_{x, y}(1)=x$. Then, $\gamma_{x, y}$ is said to possess the property (P) with respect to $y, x \in M$ if

$$
\gamma_{x, y}^{\prime}(s)(t-s)=\alpha\left(\gamma_{x, y}(t), \gamma_{x, y}(s)\right) \eta\left(\gamma_{x, y}(t), \gamma_{x, y}(s)\right), \forall s, t \in[0,1] .
$$

Remark 4.1. If $\alpha=1$, then the above property reduces to the property defined by Pini [14].

Let $M$ be a Riemannian manifold and $\gamma_{x, y}$ possessing the property (P) with respect to $y, x \in M$, then

$$
\alpha(x, y) \eta(x, y)=\alpha\left(\gamma_{x, y}(1), \gamma_{x, y}(0)\right) \eta\left(\gamma_{x, y}(1), \gamma_{x, y}(0)\right)=\gamma_{x, y}^{\prime}(0) .
$$

the case when $\gamma_{x, y}$ is a geodesic, then

$$
\begin{aligned}
\alpha\left(\gamma_{x, y}(0), \gamma_{x, y}(s)\right) \eta\left(\gamma_{x, y}(0), \gamma_{x, y}(s)\right) & =-s \gamma_{x, y}^{\prime}(s) \\
& =-s P_{0, \gamma_{x, y}}^{s}\left[\gamma_{x, y}^{\prime}(0)\right] \\
& =-s P_{0, \gamma_{x, y}}^{s}[\alpha(x, y) \eta(x, y)],
\end{aligned}
$$

or

$$
P_{s, \gamma_{x, y}}^{0}\left[\alpha\left(y, \gamma_{x, y}(s)\right) \eta\left(y, \gamma_{x, y}(s)\right)\right]=-s \alpha(x, y) \eta(x, y)
$$

and

$$
\begin{aligned}
\alpha\left(\gamma_{x, y}(1), \gamma_{x, y}(s)\right) \eta\left(\gamma_{x, y}(1), \gamma_{x, y}(s)\right) & =(1-s) \gamma_{x, y}^{\prime}(s) \\
& =(1-s) P_{0, \gamma_{x, y}}^{s}\left[\gamma_{x, y}^{\prime}(0)\right] \\
& =(1-s) P_{0, \gamma_{x, y}}^{s}[\alpha(x, y) \eta(x, y)]
\end{aligned}
$$

or

$$
P_{s, \gamma_{x, y}}^{0}\left[\alpha\left(x, \gamma_{x, y}(s)\right) \eta\left(x, \gamma_{x, y}(s)\right)\right]=(1-s) \alpha(x, y) \eta(x, y) .
$$


Hence

$$
\left.\begin{array}{l}
\left(C_{1}\right) P_{s, \gamma_{x, y}}^{0}\left[\alpha\left(y, \gamma_{x, y}(s)\right) \eta\left(y, \gamma_{x, y}(s)\right)\right]=-s \alpha(x, y) \eta(x, y), \\
\left(C_{2}\right) P_{s, \gamma_{x, y}}^{0}\left[\alpha\left(x, \gamma_{x, y}(s)\right) \eta\left(x, \gamma_{x, y}(s)\right)\right]=(1-s) \alpha(x, y) \eta(x, y)
\end{array}\right\}
$$

for all $s \in[0,1]$, which together called condition $(C)$.

Theorem 4.1. Let $M$ be a Riemannian manifold and $S$ be an open subset of $M$ which is geodesic $\alpha$-invex set with respect to $\eta: M \times M \rightarrow T M$ and $\alpha: M \times M \rightarrow$ $R \backslash\{0\}$. Let $f: S \rightarrow R$ be a differentiable and geodesic $\alpha$-preinvex function on $S$. Then, $f$ is an $\alpha$-invex function on $S$.

Proof. Since $S$ is geodesic $\alpha$-invex set with respect to $\eta$ and $\alpha$ for all $x, y \in S$, there exists a unique geodesic $\gamma_{x, y}:[0,1] \rightarrow M$ such that

$$
\gamma_{x, y}(0)=y, \gamma_{x, y}^{\prime}(0)=\alpha(x, y) \eta(x, y), \gamma_{x, y}(t) \in S, \text { for all } t \in[0,1] .
$$

But $f$ is geodesic $\alpha$-preinvex for $t \in(0,1)$, we have

$$
f\left(\gamma_{x, y}(t)\right) \leq t f(x)+(1-t) f(y),
$$

or

$$
f\left(\gamma_{x, y}(t)\right)-f(y) \leq t(f(x)-f(y))
$$

On dividing by $t$,

$$
\frac{1}{t}\left[f\left(\gamma_{x, y}(t)\right)-f(y)\right] \leq f(x)-f(y) .
$$

Taking the limit as $t \rightarrow 0$, we have

$$
d f_{\gamma_{x, y(0)}}\left(\gamma_{x, y}^{\prime}(0)\right) \leq f(x)-f(y) .
$$

Therefore,

$$
d f_{y}(\alpha(x, y) \eta(x, y)) \leq f(x)-f(y) .
$$

Hence, $f$ is $\alpha$-invex on $S$.

Theorem 4.2. Let $M$ be a Riemannian manifold and $S$ be an open subset of $M$, which is geodesic $\alpha$-invex set with respect to $\eta: M \times M \rightarrow T M$ and $\alpha: M \times M \rightarrow$ $R \backslash\{0\}$. Let $f: S \rightarrow R$ be a differentiable function and $\eta$ satisfies the condition $(C)$. Then $f$ is geodesic $\alpha$-preinvex on $S$ if $f$ is $\alpha$-invex on $S$.

Proof. We know that for geodesic $\alpha$-invex set with respect to $\eta$ and $\alpha$ for every $x, y \in S$, there exists a unique geodesic $\gamma_{x, y}:[0,1] \rightarrow M$ such that

$$
\gamma_{x, y}(0)=y, \gamma_{x, y}^{\prime}(0)=\alpha(x, y) \eta(x, y), \gamma_{x, y}(t) \in S, \text { for all } t \in[0,1] .
$$


Fix $t \in[0,1]$ and set $\bar{x}:=\gamma_{x, y}(t)$, then by geodesic $\alpha$-invexity of $f$ on $S$, we have

$$
\begin{aligned}
& f(x)-f(\bar{x}) \geq d f_{\bar{x}}(\alpha(x, \bar{x}) \eta(x, \bar{x})), \\
& f(y)-f(\bar{x}) \geq d f_{\bar{x}}(\alpha(y, \bar{x}) \eta(y, \bar{x})) .
\end{aligned}
$$

On multiplying (1) by $t$ and (2) by $(1-t)$, respectively, and then adding we get

(3) $\quad t f(x)+(1-t) f(y)-f(\bar{x}) \geq d f_{\bar{x}}[t \alpha(x, \bar{x}) \eta(x, \bar{x})+(1-t) \alpha(y, \bar{x}) \eta(y, \bar{x})]$.

By the condition $(C)$, we have

$$
\begin{aligned}
t \alpha(x, \bar{x}) \eta(x, \bar{x}) & +(1-t) \alpha(y, \bar{x}) \eta(y, \bar{x})=t(1-t) P_{0, \gamma}^{t}[\alpha(x, y) \eta(x, y)] \\
& -(1-t) t P_{0, \gamma}^{t}[\alpha(x, y) \eta(x, y)] \\
& =0 .
\end{aligned}
$$

This together with (3) implies

$$
t f(x)+(1-t) f(y) \geq f(\bar{x}) .
$$

Hence, $f$ is geodesic $\alpha$-preinvex on $S$.

\section{Geodesic $\alpha$-Preinvexity and Semicontinuity}

In this section, we discuss $\alpha$-preinvexity on Riemannian manifold under proximal subdifferential of a lower semicontinuous function. First, we recall the definition of a proximal subdifferential of a function defined on a Riemannian manifold in [5].

Definition 5.1. Let $M$ be a Riemannian manifold and $f: M \rightarrow(-\infty, \infty]$ be a lower semicontinuous function. A point $\zeta \in T_{y} M$ is said to be a proximal subgradient of $f$ at $y \in \operatorname{dom}(f)$, if there exist positive numbers $\delta$ and $\sigma$ such that

$$
f(x) \geq f(y)+\left\langle\zeta, \exp _{y}^{-1} x\right\rangle_{y}-\sigma d^{2}(x, y), \text { for all } x \in B(y, \delta),
$$

where $\operatorname{dom}(f):=\{x \in M: f(x)<\infty\}$.

The set of all proximal subgradients of $f$ at $y \in M$ is denoted by $\partial_{p} f(y)$ and is called the proximal subdifferential of $f$ at $y$.

Theorem 5.1. Let $M$ be a Riemannian manifold and $S$ be an open subset of $M$, which is geodesic $\alpha$-invex with respect to $\eta: M \times M \rightarrow T M$ and $\alpha: M \times M \rightarrow$ $R \backslash\{0\}$. Let $f: S \rightarrow R$ be geodesic $\alpha$-preinvex. If $\bar{x} \in S$ is a local minimum of the problem

(P) Minimize $f(x)$

subject to $x \in S$, 
then $\bar{x}$ is a global minimum of $(P)$.

Proof. Let $\bar{x} \in S$ be a local minimum. Then, there exists a neighborhood $N_{\epsilon}(\bar{x})$ such that

$$
f(\bar{x}) \leq f(x), \text { for all } x \in S \cap N_{\epsilon}(\bar{x}) .
$$

If $\bar{x}$ is not a global minimum of $f$, then there exists a point $x^{*} \in S$ such that

$$
f\left(x^{*}\right)<f(\bar{x}) .
$$

As $S$ is a geodesic $\alpha$-invex set with respect to $\eta$ and $\alpha$, there exists a unique geodesic $\gamma$ such that

$$
\gamma(0)=\bar{x}, \gamma^{\prime}(0)=\alpha\left(x^{*}, \bar{x}\right) \eta\left(x^{*}, \bar{x}\right), \gamma(t) \in S, \text { for all } t \in[0,1] .
$$

If we choose $\epsilon>0$ such that $d(\gamma(t), \bar{x})<\epsilon$, then $\gamma(t) \in N_{\epsilon}(\bar{x})$. From the geodesic $\alpha$-preinvexity of $f$, we have

$$
f(\gamma(t)) \leq t f\left(x^{*}\right)+(1-t) f(\bar{x})<f(\bar{x}), \text { for all } t \in(0,1) .
$$

Therefore, for each $\gamma(t) \in S \cap N_{\epsilon}(\bar{x}), f(\gamma(t))<f(\bar{x})$, which is a contradiction to (5).

Theorem 5.2. Let $M$ be a Cartan-Hadamard manifold and $S$ be an open subset of $M$, which is geodesic $\alpha$-invex with respect to $\eta: M \times M \rightarrow T M$ and $\alpha: M \times M \rightarrow$ $R \backslash\{0\}$ with $\alpha(x, y) \eta(x, y) \neq 0$ for all $x \neq y$. Assume that $f: S \rightarrow(-\infty, \infty]$ is a lower semicontinuous geodesic $\alpha$-preinvex function and $y \in \operatorname{dom}(f), \zeta \in \partial_{p} f(y)$. Then there exists a positive number $\delta$ such that

$$
f(x) \geq f(y)+\langle\zeta, \alpha(x, y) \eta(x, y)\rangle_{y}, \text { for all } x \in S \cap B(y, \delta) .
$$

Proof. From the definition of $\partial_{p} f(y)$, there are positive numbers $\delta$ and $\sigma$ such that

$$
f(x) \geq f(y)+\left\langle\zeta, \exp _{y}^{-1} x\right\rangle_{y}-\sigma d^{2}(x, y) \text {, for all } x \in B(y, \delta) .
$$

Now, fix $x \in S \cap B(y, \delta)$. Since $S$ is a geodesic $\alpha$-invex set with respect to $\eta$ and $\alpha$, there exists a unique geodesic $\gamma_{x, y}:[0,1] \rightarrow M$ such that

$$
\gamma_{x, y}(0)=y, \gamma_{x, y}^{\prime}(0)=\alpha(x, y) \eta(x, y), \gamma_{x, y}(t) \in S, \text { for all } t \in[0,1] .
$$

Given that $M$ is a Cartan-Hadamard manifold, then $\gamma_{x, y}(t)=\exp _{y}(t \alpha(x, y) \eta(x, y))$ for each $t \in[0,1]$ (see [9, p. 253]). If we choose $t_{0}=\frac{\delta}{\|\alpha(x, y) \eta(x, y)\|_{y}}$, then $\exp _{y}(t \alpha(x, y)$ $\eta(x, y)) \in S \cap B(y, \delta)$ for all $t \in\left(0, t_{0}\right)$.

From the geodesic $\alpha$-preinvexity of $f$, we get

$$
f\left(\exp _{y}(t \alpha(x, y) \eta(x, y))\right) \leq t f(x)+(1-t) f(y) \text { for all, } t \in\left(0, t_{0}\right) .
$$


Using (7) for each $t \in\left(0, t_{0}\right)$, we get

$$
\begin{aligned}
f\left(\exp _{y}(t \alpha(x, y) \eta(x, y))\right) \geq & f(y)+\left\langle\zeta, \exp _{y}^{-1} \exp _{y}(t \alpha(x, y) \eta(x, y))\right\rangle_{y} \\
& -\sigma d^{2}\left(\exp _{y}(t \alpha(x, y) \eta(x, y), y)\right. \\
= & f(y)+\langle\zeta, t \alpha(x, y) \eta(x, y)\rangle_{y} \\
& -\sigma d^{2}\left(\exp _{y}(t \alpha(x, y) \eta(x, y), y) .\right.
\end{aligned}
$$

Since $M$ is a Cartan-Hadamard manifold, for each $t \in\left(0, t_{0}\right)$, we have

$$
d^{2}\left(\exp _{y}(t \alpha(x, y) \eta(x, y), y)=\|t \alpha(x, y) \eta(x, y)\|_{y}^{2}=t^{2}\|\alpha(x, y) \eta(x, y)\|_{y}^{2}\right.
$$

Thus, from (8) and (9), we have

$$
\begin{aligned}
t f(x)+(1-t) f(y) & \geq f\left(\exp _{y}(t \alpha(x, y) \eta(x, y))\right) \\
& \geq f(y)+\langle\zeta, t \alpha(x, y) \eta(x, y)\rangle_{y}-\sigma t^{2}\|\alpha(x, y) \eta(x, y)\|_{y}^{2} .
\end{aligned}
$$

Hence

$$
t(f(x)-f(y)) \geq t\langle\zeta, \alpha(x, y) \eta(x, y)\rangle_{y}-\sigma t^{2}\|\alpha(x, y) \eta(x, y)\|_{y}^{2}
$$

Dividing by $t$ we obtain

$$
f(x)-f(y) \geq\langle\zeta, \alpha(x, y) \eta(x, y)\rangle_{y}-\sigma t\|\alpha(x, y) \eta(x, y)\|_{y}^{2} .
$$

Taking limit as $t \rightarrow 0$, we obtain

$$
f(x)-f(y) \geq\langle\zeta, \alpha(x, y) \eta(x, y)\rangle_{y}
$$

Note that $x \in S \cap B(y, \delta)$ is arbitrary. Then relation (6) holds for all $x \in S \cap B(y, \delta)$.

Corollary 5.1. Let $M$ be a Cartan-Hadamard manifold and $S$ be an open subset of $M$ which is geodesic $\alpha$-invex set with respect to $\eta: M \times M \rightarrow T M$ and $\alpha$ : $M \times M \rightarrow R \backslash\{0\}$. Assume that $f: S \rightarrow R$ be a lower semicontinuous geodesic $\alpha$-preinvex function. Let $y \in S$ and $0 \in \partial_{p} f(y)$. Then, $y$ is a global minimum of $f$.

\section{Mean Value Inequality and Mean Value Theorem}

In this section, we introduce mean value inequality and mean value theorem for Cartan-Hadamard manifold which are the extension of the results proved by Antczak [1].

Definition 6.1. Let $S$ be a nonempty subset of a Riemannian manifold $M$, which is geodesic $\alpha$-invex set with respect to $\eta: M \times M \rightarrow T M$ and $\alpha: M \times M \rightarrow R \backslash\{0\}$; 
and $x$ and $u$ be two arbitrary points of $S$. Let $\gamma:[0,1] \rightarrow M$ be the unique geodesic such that

$$
\gamma(0)=u, \gamma^{\prime}(0)=\alpha(x, u) \eta(x, u), \gamma(t) \in S, \text { for all } t \in[0,1] .
$$

A set $P_{u v}$ is said to be a closed $\eta$-path joining the points $u$ and $v:=\gamma(1)$, if

$$
P_{u v}:=\{y: y=\gamma(t) ; t \in[0,1]\} .
$$

An open $\eta$-path joining the points $u$ and $v$ is a set of the form

$$
P_{u v}^{0}:=\{y: y=\gamma(t) ; t \in(0,1)\} .
$$

If $u=v$ we set $P_{u v}^{0}:=\emptyset$.

Example 6.1. Suppose that $M$ is a Cartan-Hadamard manifold and $S$ is a geodesic $\alpha$-invex set with respect to $\eta$ and $\alpha$ defined in Ex 3.2. Let $x$ and $u$ be two arbitrary points of $S$ and $\gamma(t):=\exp _{u}(t \alpha(x, u) \eta(x, u))$. Then, for $u \in S_{1}, x \in S_{2}$ and $\alpha(x, u)=1$, we have $v:=\gamma(1)=P_{S_{1}}(x)$, and $P_{u v}$ is the unique geodesic with end points $u$ and $P_{S_{1}}(x)$.

Theorem 6.1. (Mean value inequality). Let $M$ be a Cartan-Hadamard manifold and $S$ be an open subset of $M$, which is geodesic $\alpha$-invex set with respect to $\eta$ : $M \times M \rightarrow T M$ and $\alpha: M \times M \rightarrow R \backslash\{0\}$ such that $\alpha(a, b) \eta(a, b) \neq 0$ for all $a, b \in S, a \neq b$. Let $\gamma_{b, a}(t)=\exp _{a}(t \alpha(b, a) \eta(b, a))$ for every $a, b \in S, t \in[0,1]$ and $c=\gamma_{b, a}(1)$. Then, a necessary and sufficient condition for a function $f: S \rightarrow R$ to be geodesic $\alpha$-preinvex is that the inequality

$$
f(x) \leq f(a)+\frac{f(b)-f(a)}{\alpha(b, a)\langle\eta(b, a), \eta(b, a)\rangle_{a}}\left\langle\exp _{a}^{-1} x, \eta(b, a)\right\rangle_{a}
$$

is true for all $x \in P_{c a}$.

Proof. Let $f: S \rightarrow R$ be a geodesic $\alpha$-preinvex function, $a, b \in S$ and $x \in P_{c a}$. If $x=a$ or $x=c$ then (10) is true trivially. If $x \in P_{c a}^{0}$, then $x:=\exp _{a}(t \alpha(b, a) \eta(b, a))$, for some $t \in(0,1)$. From the geodesic $\alpha$-invexity of $S$, we have $x \in S$ and

$$
t=\frac{\left\langle\exp _{a}^{-1} x, \alpha(b, a) \eta(b, a)\right\rangle_{a}}{\langle\alpha(b, a) \eta(b, a), \alpha(b, a) \eta(b, a)\rangle_{a}}=\frac{\left\langle\exp _{a}^{-1} x, \eta(b, a)\right\rangle_{a}}{\alpha(b, a)\langle\eta(b, a), \eta(b, a)\rangle_{a}} .
$$

Since $f$ is geodesic $\alpha$-preinvex on $S$, it follows that

$$
\begin{aligned}
f(x) & =f\left(\exp _{a}(t \alpha(b, a) \eta(b, a))\right. \\
& \leq t f(b)+(1-t) f(a) \\
& =f(a)+t[f(b)-f(a)] \\
& =f(a)+\frac{f(b)-f(a)}{\alpha(b, a)\langle\eta(b, a), \eta(b, a)\rangle_{a}}\left\langle\exp _{a}^{-1} x, \eta(b, a)\right\rangle_{a} .
\end{aligned}
$$


For sufficiency suppose that the mean value inequality (10) is true. Let $a, b \in S$ and $x:=\exp _{a}(t \alpha(b, a) \eta(b, a))$, for some $t \in[0,1]$. Then $x \in S$, and we have

$$
\begin{aligned}
f(x) & =f\left(\exp _{a}(t \alpha(b, a) \eta(b, a))\right) \\
& \leq f(a)+\frac{f(b)-f(a)}{\alpha(b, a)\langle\eta(b, a), \eta(b, a)\rangle_{a}}\left\langle\exp _{a}^{-1}\left(\exp _{a}(t \alpha(b, a) \eta(b, a)), \eta(b, a)\right\rangle_{a}\right. \\
& =f(a)+\frac{f(b)-f(a)}{\alpha(b, a)\langle\eta(b, a), \eta(b, a)\rangle_{a}} t \alpha(b, a)\langle(\eta(b, a)), \eta(b, a)\rangle_{a} \\
& =f(a)+t[f(b)-f(a)] \\
& =t f(b)+(1-t) f(a),
\end{aligned}
$$

which shows that $f$ is a geodesic $\alpha$-preinvex function on $S$.

Theorem 6.2. (Mean value theorem). Let $M$ be a Cartan-Hadamard manifold and $S$ be an open subset of $M$, which is nonempty, open geodesic $\alpha$-invex set with respect to $\eta: M \times M \rightarrow T M$ and $\alpha: M \times M \rightarrow R \backslash\{0\}$. Let $f: S \rightarrow R$ be differentiable on $S$. Then, for every $a, b \in S$ there exists $c \in P_{a b}^{0}$ such that

$$
f\left(\exp _{a}(\alpha(b, a) \eta(b, a))\right)-f(a)=d f_{c}\left[\left(d \exp _{a}\right)_{u}(\alpha(b, a) \eta(b, a))\right]
$$

where $u:=t_{0} \alpha(b, a) \eta(b, a), t_{0} \in(0,1)$ and $\left(d \exp _{a}\right)_{u}: T_{u}\left(T_{a} M\right) \cong T_{a} M \rightarrow T_{c} M$ is differential of $\exp _{a}$ at $u$.

Proof. Let us define the function $g:[0,1] \rightarrow R$ as follows

$$
g(t):=f\left(\exp _{a}(t \alpha(b, a) \eta(b, a))\right)-f(a)-t\left[f\left(\exp _{a}(\alpha(b, a) \eta(b, a))\right)-f(a)\right] .
$$

Since $g(1)=g(0)=0$, using Rolle's theorem, it follows that there exists $t_{0} \in(0,1)$ such that $g \prime\left(t_{0}\right)=0$. Let $c:=\exp _{a}\left(t_{0} \alpha(b, a) \eta(b, a)\right)$, then from (11) we have

$$
0=g \prime\left(t_{0}\right)=d f_{c}\left[\left(d \exp _{a}\right)_{u}(\alpha(b, a) \eta(b, a))\right]-f\left(\exp _{a}(\alpha(b, a) \eta(b, a))\right)+f(a) .
$$

or

$$
f\left(\exp _{a}(\alpha(b, a) \eta(b, a))\right)-f(a)=d f_{c}\left[\left(d \exp _{a}\right)_{u}(\alpha(b, a) \eta(b, a))\right] .
$$

Since $t_{0} \in(0,1)$, it follows from the definition that $c \in P_{a b}^{0}$, and the proof is complete.

\section{REFERENCES}

1. T. Antczak, Mean value in invexity analysis, Nonlinear Anal., 60 (2005), 1471-1484.

2. D. Azagra, J. Ferrera and F. Lopez-Mesas, Nonsmooth analysis and Hamilton-Jacobi equation on Riemannian manifolds, J. Funct. Anal., 220 (2005), 304-361. 
3. A. Barani and M. R. Pouryayevali, Invex sets and preinvex functions on Riemannian manifolds, J. Math. Anal. Appl., 328 (2007), 767-779.

4. M. Ferrara and S. Mititelu, Mond-Weir duality in vector programming with generalized invex functions on differentiable manifolds, Balkan J. Geom. Appl., 11 (2006), 80-87.

5. O. P. Ferreira, Proximal subgradient and characterization of Lipschitz function on Riemannian manifolds, J. Math. Anal. Appl., 313 (2006), 587-597.

6. O. P. Ferreira, P. R. Oliveira, Proximal point algorithm on Riemannian manifolds, Optimization, 51 (2002), 257-270.

7. M. A. Hanson, On sufficiency of the Kuhn-Tucker conditions, J. Math. Anal. Appl., 80 (1981), 545-550.

8. V. Jeyakumar, Strong and weak invexity in mathematical programming, Math. Oper. Res., 55 (1985), 109-125.

9. S. Lang, Fundamentals of Differential Geometry, Graduate Texts in Mathematics, Springer, New York, 1999.

10. C. Li, B. S. Mordukhovich, J. Wang and J. C. Yao, Weak sharp minima of Riemannian manifolds, Siam J. Optim., (Accepted).

11. S. Mititelu, Generalized invexity and vector optimization on differentiable manifolds, Diff. Geom. Dynam. Syst., 3 (2001), 21-31.

12. M. A. Noor, On generalized preinvex functions and monotonocities, J. Ineq. Pure Appl. Math., 110 (2004), 1-9.

13. M. A. Noor and K. I. Noor, Some characterizations of strongly preinvex functions, $J$. Math. Anal. Appl., 316 (2006), 697-706.

14. R. Pini, Convexity along curves and invexity, Optimization, 29 (1994), 301-309.

15. T. Rapcsak, Smooth Nonlinear Optimization in $R^{n}$, Kluwer Academic, 1997.

16. C. Udriste, Convex Functions and Optimization Methods on Riemannian Manifolds, Math. Appl., Kluwer Academic, 1994, p. 297.

R. P. Agarwal

Department of Mathematics and Statistics

King FahdUniversity of Petroleum and Minerals

P. O. Box 728, Dhahran 31261

Saudi Arabia

Permanant Address:

Department of Mathematics,

Texas A\&M University - Kingsville

700 University Blvd.

Kingsville, TX 78363-8202

U.S.A.

E-mail: agarwal@tamuk.edu 


\section{Ahmad}

Department of Mathematics and Statistics

King FahdUniversity of Petroleum and Minerals

P. O. Box 728, Dhahran 31261

Saudi Arabia

Permanant Address:

Department of Mathematics

Aligarh Muslim University

Aligarh-202 002

India

E-mail: drizhar@kfupm.edu.sa

Akhlad Iqbal

Department of Mathematics

BITS Pilani Hyderabad Compus

Hyderabad, AP-500078

India

E-mail: akhlad@bits-hyderabad.ac.in

Shahid Ali

Department of Mathematics

Aligarh Muslim University

Aligarh-202 002

India

E-mail: shahid_rrs@yahoo.co.in 